María Cristina López Areiza

Estudo das características de moduladores de amplitude fabricados com estruturas semicondutoras InAIAs/InGaAs e AlGaAs/GaAs MQW.

Tese apresentada como requisito parcial para obtenção do título de Doutor pelo Programa de Pós-Graduação em Engenharia Elétrica da PUC-Rio.

Orientadora: Patrícia Lustoza de Souza 
María Cristina López Areiza

\section{Estudo das características de moduladores de amplitude fabricados com estruturas semicondutoras InAIAs/InGaAs e AlGaAs/GaAs MQW.}

Tese apresentada como requisito parcial para obtenção do título de Doutor pelo Programa de Pós-Graduação em Engenharia Elétrica da PUC-Rio.

Prof $^{a}$. Patrícia Lustoza de Souza

Orientadora

PUC-Rio

Prof. Jean Pierre von der Weid

PUC-Rio

Prof. Wagner Nunes Rodrigues

UFMG
Prof ${ }^{a}$. Sandra Marcela Landi LabSem-CETUC-PUC

PUC-Rio

Prof. Mauricio Pamplona Pires UFRJ

Prof. Gustavo Soares Vieira IFAV-CTA

Prof $^{\mathrm{a}}$. José Eugenio Leal Coordenador Setorial do Centro

Técnico Científico - PUC-Rio 
Todos os direitos reservados. É proibida a reprodução total ou parcial do trabalho sem autorização da universidade, do autor e do orientador.

\section{María Cristina López Areiza}

Graduou-se em Física (1995) e fez Mestrado em Física com ênfase em Instrumentação (2000) na Universidade de Antioquia (Colômbia).

Ficha Catalográfica

Areiza, María Cristina López

Estudo das características de moduladores de amplitude fabricados com estruturas semicondutoras InAlAs/InGaAs e AlGaAs/GaAs MQW; orientadora: Patricia Lustoza de Souza. - Rio de Janeiro: PUC, Departamento de Engenharia Elétrica, 2004.

$$
\text { v., } 172 \text { f.: il. ; } 29,7 \mathrm{~cm}
$$

Tese (doutorado) - Pontifícia Universidade Católica do Rio de Janeiro, Departamento de Engenharia Elétrica.

Inclui referências bibliográficas.

1. Engenharia Elétrica - Teses. 2. Moduladores. 3. BPM. 4. Eletroabsorção. 5. Guias de onda. 6. InAIAs. 7. InGaAs. 8. GaAs. 9. AIGaAs. I. Souza, Patrícia Lustoza. II. Pontifícia Universidade Católica do Rio de Janeiro. Departamento de Engenharia Elétrica . III. Título. 
A meus pais, Julio Oscar López Henao e Amanda de Jesús Areiza Peláez, A seus sonhos.

A Sergio Leon Montoya Castillo (el Tancho) pelo voto de confiança, apoio moral e sentimental 


\section{Agradecimentos}

A Deus.

A minha orientadora a Professora Patrícia Lustoza de Souza e ao professor Mauricio Pamplona Pires, pela orientação e por tudo que me ensinaram.

À PUC-Rio e o CLAF, pelos auxílios concedidos, sem os quais este trabalho não poderia ter sido realizado.

Ao meus amigos e colegas de laboratório, Sandra Marcela Landi, Christiana Villas Boas Tribuzy, Artur López, Vinicius Miranda, Francis Guastavino e Marilene.

Ao Iracildo Oliveira, pela sua atenção, orientação e estar sempre nas horas mais difíceis do trabaho.

Ao Professor Jean Pierre Von der Weid, pela sua orientação e empréstimo de diferentes aparelhos.

À minha querida Amália Regina de Oliveira, pela sua companhia e incondicional ajuda.

Ao Professor Wagner Rodrigues, pela concessão do substrato de GaAs para o crescimento das amostra de AlGaAs/GaAs.

À Professora Maria Cristina Carvalho pelo empréstimo do EDFA. Ao professor Cláudio Lenz, por me permitir usar o laser sintonizável de Titânio-Safira e ao professor Newton Frateschi, por me ensinar novas maneiras de clivar as amostras semicondutoras.

Ao Giancarlo, por me ensinar a técnica para apontar a fibra óptica e ajuda no concerto de circuitos eletrônicos.

Aos meus amigos da alma Nelson Perez, Carmelina di Santis, César Rodriguez, Gladys Adriana Quintero, Sully Milena Mejía, Marina Ocampo, Márcia e Mercia Betânia, Jaime González, Julia, e todos aqueles que de uma ou outra forma participaram deste aprendizado... por todo seu apoio.

Aos professores que participaram da Comissão examinadora. 


\section{RESUMO}

Areiza, Maria Cristina López, Lustoza de Souza, Patrícia Estudo das características de moduladores de amplitude fabricados com estruturas semicondutoras InAIAs/InGaAs e A/GaAs/GaAs. Rio de Janeiro, 2005. 172p. Tese de doutorado. Departamento de Engenharia Elétrica, Pontifícia Universidade Católica de Rio de Janeiro

No presente trabalho de tese, se faz uma avaliação de moduladores de amplitude baseados no efeito de electro-absorção. As estruturas usadas para a fabricação dos dispositivos foram estruturas com poços quânticos múltiplos de InAIAs/InGaAs e AlGaAs/GaAs. As estruturas de InAIAs/InGaAs foram projetadas para trabalhar na faixa comercial das telecomunicações $(1.55 \mu \mathrm{m})$. Por isto a importância de aperfeiçoar os parâmetros de desempenho do dispositivo, tais como Stark shift, chirp, razão de contraste, perda por inserção, entre outros. Um estudo sistemático prévio destas estruturas foi realizado por Pires [Pires,1998]. Ele propõe variar a concentração de gálio na liga para produzir uma leve tensão na estrutura e modificar desta forma as propriedades ópticas do material. O estudo de [Pires,1998] propôs uma faixa de valores para variar a concentração de gálio (entre $46 \%$ e $52 \%$ ) onde pode ser encontrada a melhor condição de operação do dispositivo. Cabe a esta tese aprofundar o estudo nesta faixa de valores, e decidir os parâmetros mais adequados para operação. No referente às estruturas de $A / G a A s / G a A s$, se toma como partida uma proposta teórica de [Batty et al, 1993], e estudada posteriormente por [Tribuzy, 2001], onde se sugere usar finas camadas de dopagem delta $(\delta)$ nos poços de GaAs para melhorar o deslocamento Stark em $87 \%$ para um campo aplicado de $40 \mathrm{kV} / \mathrm{cm}$. O dispositivo foi desenhado e fabricado, obtendo-se um valor de $78 \%$ para o mesmo campo aplicado, resultado relevante, pois é a verificação experimental de uma proposta teórica.

\section{Palavras-Chave}

Moduladores de eletroabsorção, MQW, AlGaAs/GaAs, InAlAs/InGaAs, dispositivos opto-electrônicos, campo próximo. 


\section{ABSTRACT}

Areiza, Maria Cristina López, Lustoza,de Souza Patrícia. Study of the characteristics of modulators of amplitude manufactured with semiconducting structures InAlAs/InGaAs and AlGaAs/GaAs MQW. Rio de Janeiro, 2005. 172p. PhD. Thesis. Department of Electrical Engineering, Pontifícia Universidade Católica de Rio de Janeiro

In this thesis work, is made an evaluation of modulators of amplitude based in the electrum-absorption effect. The structures used for the devices were multiple quantum wells of InAIAs/InGaAs and A/GaAs/GaAs. The structures of InAlAs/InGaAs are used to work in the commercial band of the telecommunications $(1,55 \mu \mathrm{m})$. This is the reason it is important to optimize the parameters of performance of the device, such as the Stark shift, chirp, contrast reason, insertion loss, etc. Previously, a systematic study of these structures was made by [Pires, 1998], the gallium concentration was varied to produce a strain in the structure and to modify the optic properties of the material. In the study of [Pires, 1998] considered the Gallium concentration was varied between $46 \%$ and $52 \%$ in which range the best condition to operate the device can be found. This is part of the work here presented. In this thesis this range of values was studied in more detail. For the structures of $A / G a A s / G a A s$, a theoretical proposal of [Batty et al, 1993] was experimentally investigated. It was suggested a nipi structure to use a fine delta doped $(\delta)$ in the GaAs wells, this delta doped will improve in $87 \%$ the Stark shift for a field of $40 \mathrm{KV} / \mathrm{cm}$. The device was simulated and manufactured, obtaining a value of $78 \%$ for the same field applied, this is a excellent result, because this confirm the theoretical prediction.

\section{Keywords}

Electroabsorption modulator, MQW, AlGaAs/GaAs, InAIAs/InGaAs, optolectronic devices, near field. 


\section{Sumário}

Sumário

Lista de Tabelas

iii

Listas de Figuras .......................................................................... iv

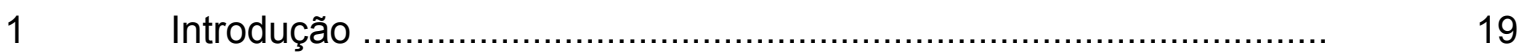

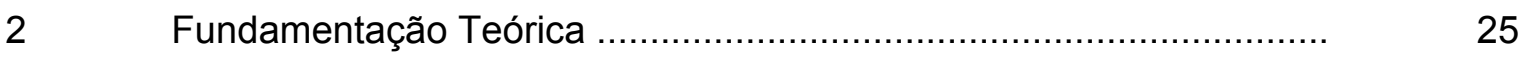

2.1 Mecanismo de absorção em poços quânticos e efeito Stark quânticr 25 confinado

2.2 Efeito Stark em estruturas de poços quânticos com dopagem delta ..

2.3 Guias de onda dielétricos

$2.4 \quad$ Propagação em guias de onda dielétricos ....................................... $\quad 34$

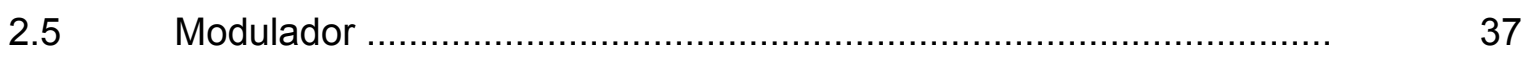

2.5.1 Características de Moduladores …............................................... 38

2.5.1.1 Razão de contraste ...............................................................

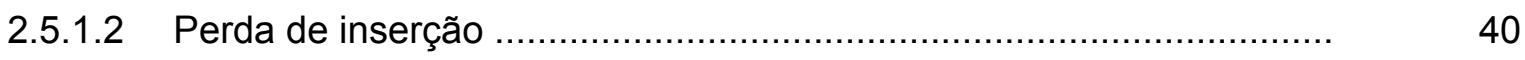

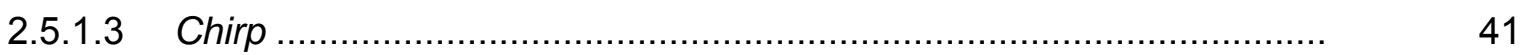

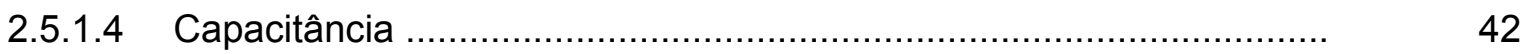

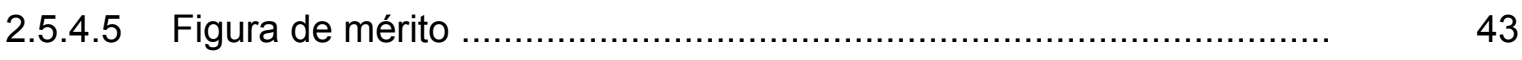

2.6 Modelo para estimar o coeficiente de absorção nas amostra: 43 AlGaAs/GaAs.

2.7 Modelo para estimar o coeficiente de absorção nas amostra: 61 InGaAs/InGaAs. 


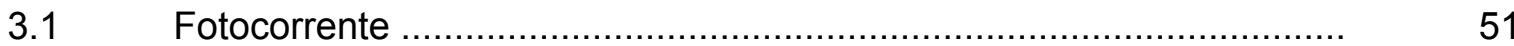

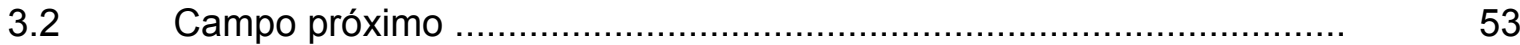

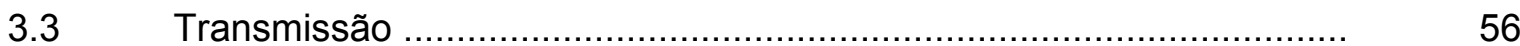

3.4 Processamento de guias de onda ................................................. 57

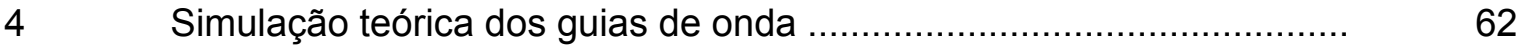

4.1 Parâmetros da simulação …...................................................... 66

4.2 Simulação da estruturas de InAIAs/InGaAs ..................................... 67

4.3 Simulação da estruturas de AlGaAs/GaAs ........................................ 72

$5 \quad$ Resultados relativos às estruturas AlGaAs/GaAs ................................

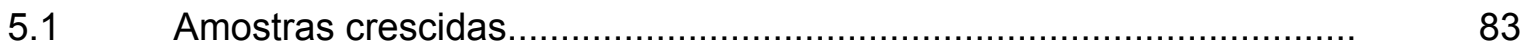

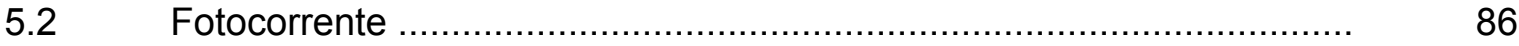

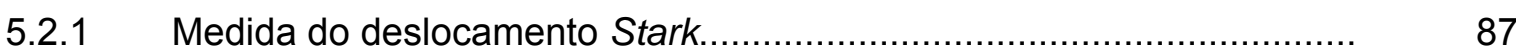

5.3 Medida de 95

5.3.1 Medida de razão de contraste......................................................... 96

$\begin{array}{lll}\text { 5.3.2 } & \text { Transmissãõ } & 99\end{array}$

$\begin{array}{lll}5.4 & \text { Medida da perda por inserçãõ } & 105\end{array}$

$\begin{array}{lll}5.5 & \text { Figuras de méritõ } & 106\end{array}$

$\begin{array}{lll}5.6 & \text { Medida do chirp̃ } & 108\end{array}$

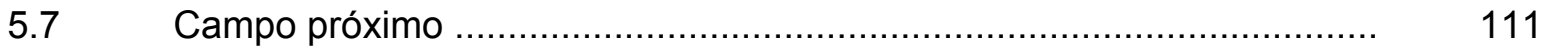

6. Resultados relativos às estruturas InAIAs/InGaAs ............................. 118

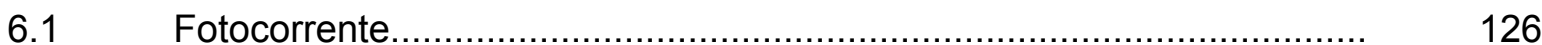


6.1.1 Deslocamento Stark ................................................................

6.1.2 Variação do coeficiente de absorção................................................... 132

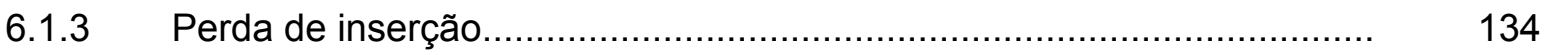

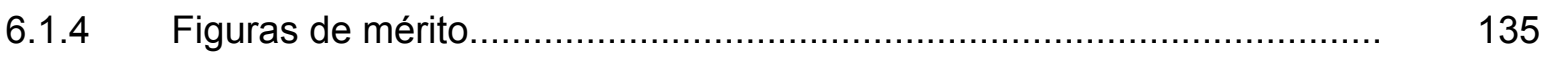

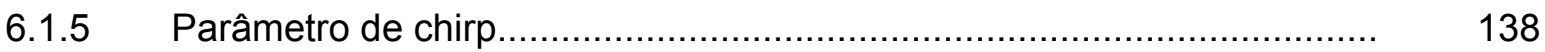

6.2. Campo próximo......................................................................... 139

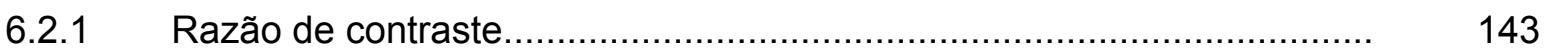

$7 \quad$ Conclusões e trabalhos futuros .................................................... 148

Referências bibliograficas.......................................................... 152

Apêndice A : Programa de campo próximo...................................... 161

Apêndice B : Programa de Fotocorrente............................................ 165

Apêndice C : Bases teórica do programa BeamProp........................... 167 


\section{Lista de Tabelas}

Tabela 4.1 - Cálculo do índice efetivo de refração da camada ativa, para diferentes valores da concentração de Alumínio

Tabela 5.1 - Propriedades estruturais das amostras de AlGaAs/GaAs estimadas a partir das medidas de raios $X$ e o modelo de massa efectiva

Tabela 5.2 - Valor do coeficiente de absorção em $\mathrm{cm}^{-1}$ medido para a geometria perpendicular, para diferentes detuning..

Tabela 5.3 - (a) Figura de mérito $(\Gamma \Delta \alpha / F)$ e (b) campo necessário para obter uma determinada razão de contraste $(10,15$ e $20 \mathrm{~dB})$ para um detuning de operação de $40 \mathrm{meV}$ para moduladores de A/GaAs/GaAs

Tabela 5.4 - Resultados do ajuste gaussiano realizado no spot do sistema de calibração

Tabela 5.5 - Medida da eficiência do acoplamento óptico em guias $(\Sigma)$ de onda com diferente largura de mesa.

Tabela 5.6 - Valores em dB da perda por propagação em guias de onda, $\mathrm{P}_{\text {guia }}$, para diferentes larguras de mesa.

Tabela 6.1 - Parâmetros estruturais da amostra 297. $T_{p}$ e $T_{b}$ são as larguras do poço e da barreira respectivamente,

Tabela 6.2 - Propriedades estruturais das amostras InAlAs/InGaAs

Tabela 6.3 - Absorção residual e perda de inserção estimadas para detuning de 30 e $40 \mathrm{meV}$

Tabela 6.4 - Campo elétrico necessário para obter 10 e $15 \mathrm{~dB}$ de razão de contraste, para um detuning de $30 \mathrm{meV}$

Tabela 6.5 - Figura de mérito $\Gamma \Delta \alpha / \Delta F$

Tabela 6.6 - Propriedades de acoplamento óptico dos guias de InAIAs/InGaAs para uma largura de mesa de $5 \mu \mathrm{m}$. 


\section{Lista de Figuras}

Figura 1.1 -Amostras com degraus dentro do poço desenvolvidas para maximizar o deslocamento Stark [Woodward et al, 1995].

Figura 2.1 - Esquema de um poço quântico típico de largura $L_{0}$ e energia de gap do material $E_{g p}$. $A$ energia do material da barreira é $E_{g b}$. A diferença de potencial das bandas de valência e condução são respectivamente $\Delta \mathrm{E}_{\mathrm{V}} \mathrm{e}$ $\Delta \mathrm{E}_{\mathrm{C}}$.

Figura 2.2 - Esquema do efeito de absorção em poços quânticos em (a) ausência e (b) presença do campo elétrico tranverso aplicado (Efeito Stark).....

Figura 2.3 - Esquema da estrutura pin utilizada para implementar experimentalmente o efeito Stark

Figura 2.4 - Esquema da dopagem delta. De acima para baixo, a primeira figura representa um plano de dopagem. O segundo gráfico é a distribuição da carga atômica e os últimos dois gráficos correspondem ao campo e ao potencial elétrico devido à dopagem

Figura 2.5 - (a) Energia potencial numa estrutura nipi em função da posição da dopagem delta. (b) Comparação do efeito da dopagem delta na estrutura de bandas. As funções de onda do estado fundamental do elétron e do buraco também estão mostradas.

Figura 2.6 - Esquema de um guia de onda planar.

Figura 2.7 - Seção transversal de estruturas de canal: a) raised strip, b) embedded strip, c) buried channel, d) guia rib, e) guia ridge. 32

Figura 2.8 - A figura (a) apresenta a geometria da estrutura utilizada por Inoue et al [Inoue et al, 1985]. Os resultados teóricos da perda por inserção estão apresentados em função: (b) da espessura da cavidade do guia e (c) da razão entre o tamanho do spot na direção vertical e o tamanho do spot na fibra utilizada para o lanzamento de luz

Figura 2.9 - Curva do fator de transmissão em função da tensão reversa aplicada. Na vizinhança do ponto $S$ do gráfico, se apresenta uma pequena linearidade que pode ser utilizada para efeito de modulação de amplitude de pequenos sinais 
Figura 2.10 - Esquema da perda de inserção e razão de contraste num modulador típico em função da diferença de potencial reversa aplicada.

Figura 2.11 - Ajuste teórico da curva de fotocorrente em função da energia para uma amostra de $A / G a A s / G a A s$, sem tensão externa aplicada.

Figura 2.12 - Espectro de transmissãode uma amostras de A/GaAs/GaAs em função do comprimento de onda em $\mu \mathrm{m}$. As linhas contínuas e tracejadas correspondem à medida experimental e ajuste teórico, respectivamente 46

Figura 2.13 - Coeficiente de absorção em função do comprimento de onda para uma amostra de $A / G a A s / G a A s$.

Figura 2.14 - Espectro de transmissão da amostra de InAIAs/InGaAs número 661 em função da energia.

Figura 2.15 - Curvas de fotocorrente e transmissão para $\Delta \mathrm{V}=0$ da amostra de InAIAs/InGaAs número 661 em unidades arbitrárias em função da energia 49

Figura 2.16 - Curva de fotocorrente para $\Delta \mathrm{V}=0$ da amostra de InAIAs/InGaAs número 661 em unidades absolutas em função da energia

Figura 3.1 - Configuração da experiência de fotocorrente.

Figura 3.2 - Geometria de lentes utilizada para o acoplamento do feixe de luz nos guias de onda

Figura 3.3 - Circuito usado para polarizar o dispositivo e cortar o sinal DC 52

Figura 3.4 - Geometria de campo próximo e de campo distante. O plano da imagem está paralelo ao plano xy. z é o eixo de propagação do campo

Figura 3.5 - Esquema da experiência usada para medir o campo próximo em guias de onda 54

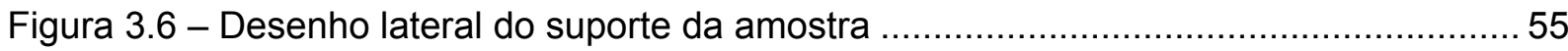

Figura 3.7 - Foto dos componentes usados na medida de campo próximo ......................... 55

Figura 3.8 - Geometria da montagem da medida da tarnsmissão...................................... 56

Figura 3.9 - Curva de calibração para corrosão de AlGaAs/GaAs ....................................... 60

Figura 3.10 - Curva de calibração para corrosão de InAIAs/InGaAs .................................. 60

Figura 3.11 - Máscaras utilizadas no processamento de guias de onda. 61

Figura 4.1 - Parâmetros geométricos considerados na simulação Beam Propagation Method (BPM) 62

Figura 4.2 - Parâmetros geométricos variados na simulação BPM. O valor do slab é zero quando a corrosão chega até o substrato. Quando não se realiza nenhuma corrosão o valor do slab é máximo, esta condição corresponde a um guia de onda planar. 
Figura 4.3 - Exemplo do computo espectral dos modos. A figura mostra potência relativa em função da constante de propagação

Figura 4.4 - Cálculo espectral dos modos confinados no guia de A/GaAs/GaAs desenhado para esta tese.

Figura 4.5 - Esquema da estrutura proposta para fabricar guias de InAIAs/InGaAs. 68

Figura 4.6 - Variação da potência total normalizada em função do slab .... 69

Figura 4.7 - Variação da potência total normalizada em função da largura de mesa do guia de onda 70

Figura 4.8 - Cálculo da potência relativa dos modos confinados no guia de onda em função da constante de propagação de cada modo 71

Figura 4.9 - Cálculo do modo fundamental TE em InAIAs/InGaAs. 71

Figura 4.10 - Perfil 3D do modo fundamental TE em guias de onda de InAIAs/InGaAs, com largura de mesa de $5 \mu \mathrm{m}$ e slab igual a zero

Figura 4.11 - Estrutura proposta por Dong et al para cavidade óptica de laser [Dong et al , 1994].

Figura 4.12 - Esquema da estrutura proposta para fabricar guias de A/GaAs/GaAs 74

Figura 4.13 - Variação da potência total relativa em função da largura de mesa para guias de onda de $A / G a A s / G a A s$ 75

Figura 4.14 - Variação da potência total relativa em função do slab para guias de onda de $A / G a A s / G a A s$. 75

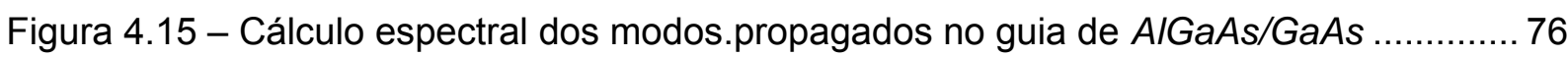

Figura 4.16 - Cálculo do modo fundamental TE em A/GaAs/GaAs ...................................... 77

Figura 4.17 - Perfil 3D do modo fundamental TE em AlGaAs/GaAs 77

Figura 5.1 - (a) Esquema que ilustra a posição da dopagem delta no poço (linha tracejada) e na barreira (linha contínua). (b) Forma do potencial depois de

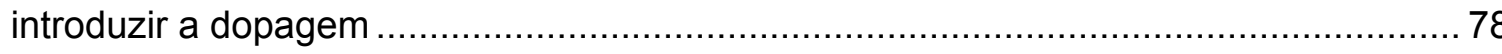

Figura 5.2 - Densidade de buracos na banda de valência em função da posição ................ 80 Figura 5.3 -Fotocorrente das amostras 713 e 714 , com dopagem $\boldsymbol{n}=+20 \% \boldsymbol{p}$ e $\boldsymbol{n}=+\% 10 \boldsymbol{p}$ respectivamemte. Medida realizada com incidência de luz perpendicular. O deslocamento Stark $(\Delta S)$ é estimado para uma diferença de potencial de 4 volts .81

Figura 5.4 - Calibração da concentração da dopagem delta em função do fluxo. 82 
Figura 5.5 - Esquema da estrutura nipi de AlGaAs/GaAs. As linhas pontilhadas indicam a posição da dopagem delta nas diferentes camadas

Figura 5.6 - Relação teórica da energia de transição e1-hh1 $\left(E_{g}\right)$ em função da largura do poço das estruturas $A / G a A s / G a A s$ estimada com o modelo de massa efetiva

Figura 5.7 - (a) Incidência da luz no plano das camadas epitaxiais, geometria usada em guias de onda e (b) Incidência da luz de forma transversal às camadas epitaxiais

Figura 5.8 - Deslocamento Stark teórico das amostras A/GaAs/GaAs em função do campo elétrico aplicado. $O$ valor $L_{p}$ que se encontra acima de cada linha corresponde ao valor da largura do poço considerado para cada cálculo

Figura 5.9 - Fotocorrente no plano das estruturas de AlGaAs/GaAs: (a) com dopagem delta e (b) sem dopagem delta. Medidas realizadas com luz despolarizada a $300 \mathrm{~K}$

Figura 5.10 - Fotocorrente na geometria no plano e perpendicular às estruturas de $A / G a A s / G a A s$ (a) sem dopagem delta e (b) com dopagem delta. Medidas realizadas com luz despolarizada a $300 \mathrm{~K}$ 90

Figura 5.11 - Medida de fotocorrente em função da energia para as diferentes geometrias, realizadas para ilustrar o efeito da polarização na estrutura A/GaAs/GaAs sem dopagem delta

Figura 5.12 - Medida de fotocorrente em função da energia para as diferentes geometrias, realizadas para ilustrar o efeito da polarização na estrutura A/GaAs/GaAs com dopagem delta

Figura 5.13 - Deslocamento Stark em função do campo elétrico aplicado para vários guias de onda de $A / G a A s / G a A s$, considerando luz despolarizada 92

Figura 5.14 - Razão entre o deslocamento Stark entre a amostra nipi de AlGaAs/GaAs e o deslocamento Stark nas amostras sem delta em função do campo elétrico aplicado

Figura 5.15 - Deslocamento Stark médio calculado a partir da fotocorrente das amostras de $A / G a A s / G a A s$ na geometria paralela em função do campo elétrico aplicado

Figura 5.16 - Deslocamento Stark em função do campo elétrico aplicado medido a partir da fotocorrente na geometria perpendicular. 95

Figura 5.17 - Razão de contraste medida em função da tensão reversa aplicada em guias com diferentes larguras de mesa e $250 \mu \mathrm{m}$ de comprimento 
Figura $5.18-\Delta \alpha$ na geometria paralela em unidades absolutas em função da tensão reversa aplicada para $A / G a A s / G a A s$, com largura de mesa $W=7 \mu \mathrm{m}$ e detuning de $40 \mathrm{meV}$.... 98

Figura 5.19 - Relação entre as energias do gap dos materiais usados para fabricar a estrutura de $A / G a A s / G a A s$. 99

Figura 5.20 - Espectros de transmissão das amostras (a) com dopagem delta e (b) referência em função do comprimento de onda em $\mu \mathrm{m}$. 100

Figura 5.21 - Janela do programa Spectrum, o qual foi utilizado para realizar o ajuste da curva teórica de fotocorrente 101

Figura 5.22 - Ajuste teórico das curvas da fotocorrente perpendicular para amostras (a) com dopagem delta e (b) sem dopagem delta em função da energia 102

Figura 5.23 - Espectros de transmissão das amostras (a) com dopagem delta e (b) referência em função do comprimento de onda em $\mu \mathrm{m}$. 103

Figura 5.24 - Coeficiente de absorção $\left(\mathrm{cm}^{-1}\right)$ em (a) amostra com dopagem delta e (b) sem dopagem delta em função do comprimento de onda $(\mu \mathrm{m})$ estimado mediante o ajuste da curva de transmissão 103

Figura $5.25-\Delta \alpha$ calculada a partir da fotocorrente perpendicular em função da tensão reversa aplicada nas amostras de A/GaAs/GaAs 104

Figura 5.26 - Fotocorrente medida na geometria paralela em função da energia.

Medida realizada com luz polarizada 105

Figura 5.27 - PI em função da tensão reversa aplicada, para detuning de 40 $\mathrm{meV}$, com um comprimento de guia de $250 \mu \mathrm{m}$ e $\Gamma=0.077$. 106

Figura 5.28 - Parâmetro de chirp calculado a partir das medidas de fotocorrente na geometria paralela e perpendicular para amostra (a) sem dopagem delta e (b) com dopagem delta em função do campo aplicado 109

Figura 5.29 - Exemplo de cálculo teórico de $\Delta \alpha$ para amostras de InGaAs/GaAs em função do comprimento de onda de operação

Figura 5.30 - Estrutura nipi proposta para fazer os moduladores de AlGaAs/GaAs com dopagem delta na camada ativa.

Figura 5.31 - Perfil gaussiano (a) teórico e (b) experimental de um guia de onda fabricado com a estrutura 819. Usa-se uma largura de mesa de $5 \mu \mathrm{m}$. 113

Figura 5.32 - Sistema de calibração do campo próximo e o tamanho do spot 113

Figura 5.33 - Fator de confinamento óptico em guias de onda em função da largura de mesa.

Figura 6.1 - Energia do gap em função do parâmetro de rede. 
Figura 6.2 - Estrutura básica das amostras InAIAs/InGaAs. Os valores $\mathrm{T}_{\mathrm{p}}$ e $\mathrm{T}_{\mathrm{b}}$

correspondem à largura do poço e da barreira, respectivamente 120

Figura 6.3 - Largura dos poços em função da concentração de gálio na liga InGaAs calculada para diferentes energias de transição

Figura 6.4 - (a) Parâmetro de chirp e (b) $\Delta \alpha$ para a amostra 297 em função do campo elétrico

Figura 6.5 - Largura do poço em função da concentração de gálio na liga InGaAs. Os números nos retângulos correspondem às amostras novas crescidas para esta tese

Figura 6.6 - Esquema da amostra 665. Para as outras amostras de InAIAs/InGaAs tais como 664, 661, 657 e 653, muda a espessura da região ativa, pois as amostras diferem nas larguras de poço e barreira

Figura 6.7 - Geometria da fotocorrente perpendicular e no plano 126

Figura 6.8 - Espectro de fotocorrente em função da energia da amostra 665, nas diferentes geometrias, para uma diferença de potencial zero

Figura 6.9 - Espectro de fotocorrente da amostra 653, nas diferentes geometrias, para uma diferença de potencial zero.

Figura 6.10 - Espectro de fotocorrente da amostra 657, nas diferentes geometrias, para uma diferença de potencial zero.

Figura 6.11 - Espectro de fotocorrente da amostra 661, nas diferentes geometrias, para uma diferença de potencial zero.

Figura 6.12 - Espectro de fotocorrente da amostra 664, nas diferentes geometrias, para uma diferença de potencial zero.

Figura 6.13 - Deslocamento Stark medido a partir da fotocorrente na geometria perpendicular de amostras de $\ln A / A s / \operatorname{InGaAs}$

Figura 6.14 - Valor de $\Delta \alpha\left(\mathrm{cm}^{-1}\right)$ em função do campo aplicado estimada a partir da fotocorrente perpendicular das amostras de InAIAs/InGaAs para um detuning de $30 \mathrm{meV}$.

Figura 6.15 - Mesmo gráfico da figura 6.5, onde os números das amostras foram substituídos pelo valor da perda por inserção para um detuning de $30 \mathrm{meV}$ 135

Figura 6.16 - Mesmo gráfico da figura 6.5, onde os números das amostras foram substituídos pelo valor do campo elétrico necessário para obter $10 \mathrm{~dB}$ de razão de contraste e detunnig de $30 \mathrm{meV}$. 136

Figura 6.17 - Mesmo gráfico da figura 6.5, onde os números das amostras foram substituídos pelo valor da figura de mérito $\Gamma \Delta \alpha / \Delta F$ para obter $10 \mathrm{~dB}$ de 
razão de contraste com um detunnig de $30 \mathrm{meV}$. A região sombreada é a região onde se obteve a melhor condição de operação do dispositivo

Figura 6.18 - Parâmetro de chirp $\left(\alpha_{\mathrm{L}}\right)$ calculado a partir das curvas de fotocorrente perpendicular das amostras de InAIAs/InGaAs e comparados com o parâmetro de chirp das estruturas 296 e 297, para um detuning de 30 $\mathrm{meV}$ 138

Figura 6.19 - Geometria da estrutura de InAIAs/InGaAs utilizada para fabricar os dispositivos desta tese. 139

Figura 6.20 - Perfil dos guias após a corrosão com (a) $W=7 \mu \mathrm{m}$ e (b) $W=3 \mu \mathrm{m}$ 140

Figura 6.21 - Imagem de MEV dos guias processados depois da deposição de polimida 140

Figura 6.22 - Perfil 3D do campo próximo do guia de onda 665 141

Figura 6.23 - Imagens da intensidade do campo (a) experimental e (b) teórico medidas na face de saída do guia

Figura 6.24 - Medida de razão de contraste nos guias de InAIAs/InGaAs em função da tensão aplicada para luz despolarizada.

Figura 6.25 - Simulação da propagação de diferentes modos existentes na cavidade óptica da estrutura mostrada na figura 6.18

Figura 6.26 - Cálculo espectral dos modos existentes na cavidade óptica da estrutura (a) 661 e (b) 665 em função da constante de propagação dos modos 